Ciencia y Educación, Vol. 6, No. 1, enero-abril, 2022

ISSN (impreso): 2613-8794・ISSN (en línea): 2613-8808

DOI: https://doi.org/10.22206/cyed.2022.v6i1.pp83-94

\title{
Actitudes docentes frente al estudiantado con discapacidad: una revisión de literatura
}

\author{
Teaching attitudes towards students with disabilities: \\ A literature review
}

Jefferson Telloa ORCID: 0000-0002-3054-4290

Recibido: 17/07/2021 • Aprobado: 11/08/2021

Cómo citar: Tello, J. Actitudes docentes frente al estudiantado con discapacidad: una revisión de literatura. Ciencia y Educación, 6(1), 83-94. https://doi.org/10.22206/cyed.2022.v6i1.pp83-94

Resumen

Esta revisión de literatura tiene el objetivo de analizar investigaciones relacionadas con las actitudes de los docentes sobre la inclusión de estudiantes con discapacidad, publicadas entre los años 2010 y 2021. La postura de los docentes con respecto a la inclusión de estudiantes con discapacidad en el aula regular se convierte en una de las condiciones fundamentales para alcanzar resultados exitosos en este proceso. Para esto se consultaron las bases de datos SciElo, Redalyc y Dialnet y el portal ResearchGate, realizando un proceso de selección tras el cual se obtuvieron 20 artículos para su estudio. Los resultados muestran que las actitudes de los docentes son positivas en su mayoría, siendo más favorables en los docentes más jóvenes. Algunas de las causas de las actitudes negativas se basan en la formación de los docentes, su poca experiencia en la atención a la diversidad y la falta de apoyo en sus instituciones ${ }^{\mathrm{b}}$.

Palabras clave: Educación inclusiva, discapacidad, actitudes docentes, inclusión, docentes.

\begin{abstract}
This literature review aims analyze research related to the attitudes of teachers about the inclusion of students with disabilities published between 2010 and 2021. The position of teachers regarding the inclusion of students with disabilities in the regular classroom becomes one of the fundamental conditions to achieve successful results in this process. For this, the SciElo, Redalyc, and Dialnet databases, and the ResearchGate portal were consulted, carrying out a selection process, obtaining 20 articles for study. The results show that teachers' attitudes are mostly positive, being more favorable in younger teachers. Some of the causes of negative attitudes are based on the training of teachers, their little experience in attending to diversity and the lack of support in their institutions.
\end{abstract}

Keywords: Inclusive education, disability, teaching attitudes, inclusion, teachers.

\footnotetext{
a Universidad Pontificia Bolivariana, Colombia. Correo-e: jefferson.tello@upb.edu.co

${ }^{\mathrm{b}}$ Este trabajo forma parte de las revisiones preliminares para establecer el estado del arte de la tesis doctoral "Actitudes de docentes oficiales de básica primaria frente a la inclusión de estudiantes con discapacidad en el aula”. Desarrollado como parte del Doctorado en Educación de la Universidad Pontificia Bolivariana sede Medellín.
} 


\section{Introducción}

La educación inclusiva es uno de los ideales de los sistemas educativos alrededor del mundo en la actualidad (Soto, 2007; Angenscheidt \& Navarrete, 2017; Beltrán et al., 2015; Amor et al., 2021), pues existe un consenso de que uno de los mecanismos más eficientes para desarrollar una sociedad justa y equitativa se origina en procesos educativos en el marco de una escuela inclusiva, diversa y de calidad (UNESCO, 1994, Sánchez-Gómez et al., 2020). En esta medida, organismos internacionales y gobiernos de varios países plantean acuerdos, leyes y decretos que propenden por el establecimiento de prácticas educativas inclusivas acordes a los derechos de todas las personas (Soto, 2007; Novo-Corti et al., 2015; Mora et al., 2020; Baez \& D’ Ottavio, 2020). En este marco, la situación de las personas con discapacidad ha cambiado debido a la resignificación de su rol en la sociedad, posibilitando así su participación en múltiples contextos (Soto, 2007; Sanhueza et al., 2012; Polo \& Aparicio, 2018; Beltrán et al., 2015; Verdugo et al., 2020; Mora et al., 2020; Córdoba-Andrade et al., 2021). Dentro de este panorama, educar en la diversidad implica reconocer las características individuales de cada sujeto, considerando que la inclusión no alude únicamente a la discapacidad, sino a todas las particularidades de los seres humanos, incluyendo factores culturales, económicos, sociales y religiosos (Bravo, 2013; Darretxe et al., 2020). Así pues, la educación inclusiva se refiere al derecho de las personas a recibir una educación de calidad que respete la diversidad y las diferencias individuales (Ministerio de Educación Nacional, 2007; González \& Triana, 2018; Latorre, 2013; Acosta \& Arráez, 2014).

Ahora bien, la materialización de las iniciativas gubernamentales con relación a los procesos de educación inclusiva, tanto nacionales como globales, recaen de forma directa sobre las instituciones educativas y sobre los docentes de manera particular, pues son ellos los encargados de acompañar a todos los estu- diantes, incluidos los que presentan alguna discapacidad (Sanhueza et al., 2012; Tárraga-Mínguez et al., 2020; Slee, 2018). De acuerdo con la Organización de las Naciones Unidas (2006), una persona tiene una discapacidad cuando debido a sus deficiencias físicas, mentales o intelectuales a largo plazo afronta dificultades para interactuar y participar en la sociedad bajo las mismas condiciones que los demás. En este sentido, los docentes y sus actitudes con respecto a la inclusión juegan un papel determinante en el desarrollo de procesos educativos exitosos, pues múltiples investigaciones han demostrado que las actitudes del profesorado repercuten directamente en la forma en que estos asumen su rol y su responsabilidad acerca de los estudiantes con discapacidad, aspecto que afecta notablemente el acceso, la permanencia y el rendimiento académico de esta población (Sanhueza et al., 2012; Polo \& Aparicio, 2018). En consonancia con lo anterior, analizar las actitudes del colectivo docente frente a los procesos de educación de estudiantes con discapacidad resulta importante, pues las actitudes de los maestros pueden ser el principal apoyo o la mayor barrera para la implementación de prácticas educativas inclusivas (Blanco, 2006; Víquez et al., 2020; Molinar \& Cervantes, 2021; Morales, 2017).

\section{Metodología}

El objetivo de este trabajo es revisar publicaciones especializadas que abordan los factores que influyen en las actitudes de los docentes hacia la inclusión de estudiantes con discapacidad, presentadas entre los años 2010 y 2021. Para la selección de los artículos objeto de revisión se utilizaron los términos de búsqueda "actitudes docentes", "actitudes docentes y discapacidad", “actitudes docentes e inclusión” y "actitudes docentes y educación inclusiva", derivados de los resultados arrojados por la plataforma Tesauros de la UNESCO. Luego, se realizó una búsqueda en las bases de datos SciElo, Redalyc y Dialnet y el portal ResearchGate. Se seleccionaron estas bases de datos pues, por su natu- 
raleza abierta, permiten el acceso de un alto número de autores y lectores, sin las restricciones que las plataformas de pago generan, contribuyendo a la democratización del conocimiento científico. Para cumplir con el objetivo de esta revisión, se plantearon unos criterios básicos para realizar la selección de los artículos y su análisis. Dentro de los criterios de inclusión se tomaron todos los artículos escritos en idioma español, producto de investigaciones realizadas en el contexto iberoamericano y que fueron publicadas entre los años 2010 y 2021, aplicando los filtros de idioma (español) y año de publicación (2010-2021). Los criterios de inclusión para los estudios son:

a) Tema: que aborden las actitudes de los docentes con relación a la inclusión de estudiantes con discapacidad en todos los niveles escolares.

b) Participantes: estudios realizados con maestros en diferentes niveles escolares.

c) Idioma: estudios redactados en idioma español.

d) Rango temporal: estudios realizados entre 2010 y 2021 .

e) Tipo de artículo: artículos completos.

En una búsqueda inicial se presentaron 143 resultados, de los cuales se realizó una lectura de los títulos, las palabras clave y los resúmenes, para seleccionar los que se adaptaban a los criterios de inclusión, al tiempo que se eliminaban los textos repetidos (19). Finalmente, 20 artículos cumplieron con todos los criterios establecidos. Entre ellos, 8 provienen de Redalyc, 5 de Scielo, 4 de ResearchGate y 3 de Dialnet. En la Figura 1 se resume el proceso de búsqueda, la selección de artículos y el posterior análisis de estos.
Figura 1

Etapas de la búsqueda de datos
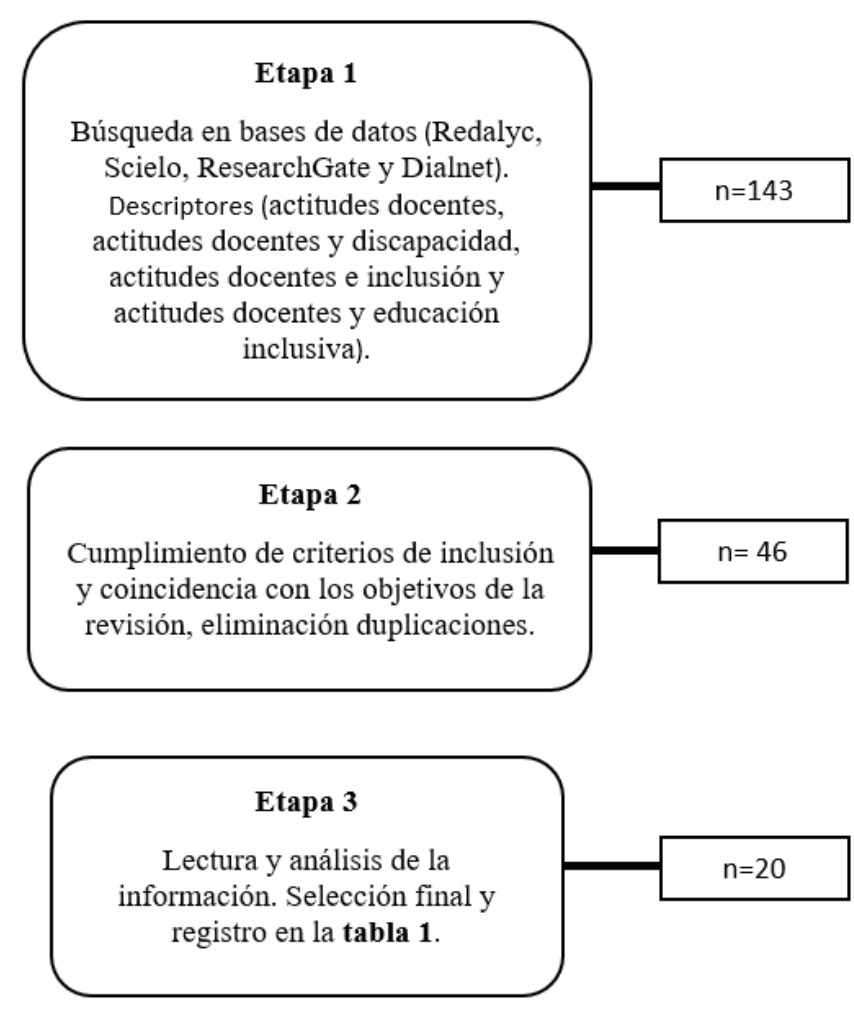

\section{Resultados}

A continuación, se detallan los resultados obtenidos tras la revisión bibliográfica realizada. En primer lugar, se presentan los trabajos con relación a su metodología, el tipo de instrumento utilizado para la recolección de información y la muestra. Esta información se presenta en la Tabla 1. Seguidamente, se analizan las publicaciones empleando un sistema de categorización que consta de 6 componentes asociados a las coincidencias temáticas observadas, a partir de unos intereses investigativos semejantes. 
Tabla 1

Organización de resultados

\begin{tabular}{|c|c|c|c|c|}
\hline Autor/Ańo & País & Objetivo & $\begin{array}{l}\text { Tipo de } \\
\text { estudio/ } \\
\text { Muestra }\end{array}$ & Medición \\
\hline $\begin{array}{l}\text { Díaz \& Franco } \\
(2010)\end{array}$ & Colombia & $\begin{array}{l}\text { Caracterizar e interpretar las actitudes y percepciones } \\
\text { hacia la inclusión educativa de los docentes de } \\
\text { instituciones educativas integradoras del municipio } \\
\text { de Soledad (Atlántico). }\end{array}$ & $\begin{array}{l}\text { Cuanti } \\
\mathrm{n}=23\end{array}$ & $\begin{array}{l}\text { Escala de actitudes } \\
\text { (EAPROF) }\end{array}$ \\
\hline $\begin{array}{l}\text { Sanhueza, } \\
\text { Granada \& } \\
\text { Bravo (2012) }\end{array}$ & Chile & $\begin{array}{l}\text { Identificar las medidas que favorecen la educación } \\
\text { inclusiva en ambos colectivos de profesorado. }\end{array}$ & $\begin{array}{l}\text { Cuanti } \\
\mathrm{n}=218\end{array}$ & $\begin{array}{l}\text { Escala de Actitudes hacia las } \\
\text { Personas con Discapacidad } \\
\text { (EAPD) }\end{array}$ \\
\hline $\begin{array}{l}\text { Acosta \& Arráez } \\
(2014)\end{array}$ & Venezuela & $\begin{array}{l}\text { Determinar las actitudes del docente de educación } \\
\text { inicial y primaria que favorecen o limitan la } \\
\text { inclusión escolar de la persona con DM. }\end{array}$ & $\begin{array}{l}\text { Cuali } \\
\mathrm{n}=10\end{array}$ & $\begin{array}{l}\text { Observación participante } \\
\text { Entrevista semi estructurada }\end{array}$ \\
\hline $\begin{array}{l}\text { Montánchez } \\
\text { (2014) }\end{array}$ & Ecuador & $\begin{array}{l}\text { Saber cuáles son las actitudes, los conocimientos } \\
\text { y las prácticas ante la inclusión educativa de los } \\
\text { docentes. }\end{array}$ & $\begin{array}{l}\text { Cuanti } \\
\mathrm{n}=321\end{array}$ & $\begin{array}{l}\text { Cuestionario de elaboración } \\
\text { del autor }\end{array}$ \\
\hline Abellán (2015) & España & $\begin{array}{l}\text { Estudiar las actitudes hacia la discapacidad de los } \\
\text { futuros maestros de educación física. }\end{array}$ & $\begin{array}{l}\text { Cuanti } \\
\mathrm{n}=76\end{array}$ & $\begin{array}{l}\text { Escala de Actitudes hacia las } \\
\text { Personas con Discapacidad } \\
\text { (EAPD) }\end{array}$ \\
\hline $\begin{array}{l}\text { Novo-Corti., } \\
\text { Muñoz-Cantero } \\
\text { \& Calvo-Babío } \\
(2015)\end{array}$ & España & $\begin{array}{l}\text { Identificar los factores que determinan la actitud } \\
\text { favorable y la predisposición a ayudar de los } \\
\text { estudiantes universitarios hacia sus compañeros con } \\
\text { discapacidad. }\end{array}$ & $\begin{array}{l}\text { Cuanti } \\
\mathrm{n}=621\end{array}$ & $\begin{array}{l}\text { Encuesta elaboración de los } \\
\text { autores }\end{array}$ \\
\hline $\begin{array}{l}\text { Pegalajar \& } \\
\text { Colmenero } \\
(2017)\end{array}$ & España & $\begin{array}{l}\text { Identificar cuáles son las actitudes y el conocimiento } \\
\text { que poseen los docentes de Educación Secundaria } \\
\text { sobre la educación inclusiva. }\end{array}$ & $\begin{array}{l}\text { Cuanti } \\
\mathrm{n}=407\end{array}$ & $\begin{array}{l}\text { Cuestionario descriptivo } \\
\text { elaboración de los autores }\end{array}$ \\
\hline $\begin{array}{l}\text { Angenscheidt } \\
\text { \& Navarrete } \\
\text { (2017) }\end{array}$ & Uruguay & $\begin{array}{l}\text { Describir las actitudes de los docentes de enseñanza } \\
\text { inicial y primaria en un colegio privado de } \\
\text { Montevideo sobre la educación inclusiva. }\end{array}$ & $\begin{array}{l}\text { Cuanti } \\
\mathrm{n}=44\end{array}$ & $\begin{array}{l}\text { Escala de Opinión acerca de } \\
\text { la Educación Inclusiva }\end{array}$ \\
\hline $\begin{array}{l}\text { Fernández- } \\
\text { Rivas \& Espada- } \\
\text { Mateos (2017) }\end{array}$ & España & $\begin{array}{l}\text { Profundizar en la actitud del profesorado hacia la } \\
\text { integración de los ACNEEs. }\end{array}$ & $\begin{array}{l}\text { Cuanti } \\
\mathrm{n}=40\end{array}$ & Cuestionario \\
\hline $\begin{array}{l}\text { Polo \& Aparicio } \\
(2018)\end{array}$ & España & $\begin{array}{l}\text { Analizar las actitudes que tienen los docentes } \\
\text { de educación infantil de Granada acerca de los } \\
\text { estudiantes con necesidades educativas especiales. }\end{array}$ & $\begin{array}{l}\text { Cuanti } \\
\mathrm{n}=78\end{array}$ & $\begin{array}{l}\text { Escala de Actitudes hacia las } \\
\text { Personas con Discapacidad } \\
\text { (EAPD) }\end{array}$ \\
\hline $\begin{array}{l}\text { González Rojas } \\
\text { \& Triana-Fierro } \\
\text { (2018) }\end{array}$ & Colombia & $\begin{array}{l}\text { Revisar publicaciones especializadas que abordan los } \\
\text { factores influyentes en las actitudes de los docentes } \\
\text { hacia la inclusión de estudiantes con necesidades } \\
\text { educativas especiales. }\end{array}$ & $\begin{array}{l}\text { Artículo } \\
\text { revisión }\end{array}$ & N/A \\
\hline $\begin{array}{l}\text { Sevilla, Martín } \\
\text { \& Jenaro (2018) }\end{array}$ & México & $\begin{array}{l}\text { Analizar la actitud de los docentes hacia la educación } \\
\text { inclusiva. }\end{array}$ & $\begin{array}{l}\text { Cuanti } \\
\mathrm{n}=388\end{array}$ & Cuestionario de tipo cerrado \\
\hline $\begin{array}{l}\text { Tárraga, } \\
\text { Vélez, Pastor } \\
\text { \& Fernández } \\
(2020)\end{array}$ & Ecuador & $\begin{array}{l}\text { Analizar las actitudes hacia la inclusión educativa en } \\
\text { una muestra de } 66 \text { docentes de } 15 \text { centros educativos } \\
\text { de la ciudad de Cuenca (Ecuador). }\end{array}$ & $\begin{array}{l}\text { Cuanti } \\
\mathrm{n}=66\end{array}$ & $\begin{array}{l}\text { Adaptación de la escala } \\
\text { ORI: Opinions Relative to } \\
\text { Integration ofnStudents with } \\
\text { Disabilities Scale }\end{array}$ \\
\hline $\begin{array}{l}\text { Solís \& Borja } \\
(2020)\end{array}$ & Colombia & $\begin{array}{l}\text { Analizar de manera sistemática las publicaciones } \\
\text { concernientes a la actitud hacia la discapacidad del } \\
\text { profesorado de EF. }\end{array}$ & $\begin{array}{l}\text { Artículo } \\
\text { Revisión }\end{array}$ & N/A \\
\hline
\end{tabular}




\begin{tabular}{lllll}
\hline $\begin{array}{l}\text { Bermúdez } \\
\text { \& Navarrete } \\
(2020)\end{array}$ & Uruguay & $\begin{array}{l}\text { Conocer las actitudes de los docentes con la } \\
\text { finalidad de entender su predisposición para actuar } \\
\text { y realizar una propuesta de intervención en aula que } \\
\text { atienda la diversidad. }\end{array}$ & $\begin{array}{l}\text { Cuanti } \\
\mathrm{n}=42\end{array}$ & $\begin{array}{l}\text { Escala de Actitudes hacia las } \\
\text { Personas con Discapacidad } \\
\text { (EAPD) }\end{array}$ \\
\hline $\begin{array}{l}\text { Molinar \& } \\
\begin{array}{l}\text { Cervantes } \\
(2020)\end{array}\end{array}$ & Argentina & $\begin{array}{l}\text { Analizar y comparar los conocimientos y las } \\
\text { actitudes de los profesores de educación básica hacia } \\
\text { el alumnado con TDAH en Argentina y México. }\end{array}$ & $\begin{array}{l}\text { Cuanti } \\
\mathrm{n}=48\end{array}$ & ASKAT \\
\hline $\begin{array}{l}\text { Víquez et al. } \\
(2020)\end{array}$ & Costa Rica & $\begin{array}{l}\text { Observar el efecto de un programa multideportivo } \\
\text { inclusivo sobre las actitudes de niños, nińas, jóvenes, } \\
\text { padres, madres y personal de instrucción hacia las } \\
\text { personas con discapacidad. }\end{array}$ & $\begin{array}{l}\text { Cuanti } \\
\mathrm{n}=33\end{array}$ & $\begin{array}{l}\text { Escala Actitudes hacia las } \\
\text { Personas con Discapacidad } \\
\text { (EAPD) }\end{array}$ \\
\hline $\begin{array}{l}\text { Hernández \& } \\
\text { Marchesi }(2021)\end{array}$ & España & $\begin{array}{l}\text { Conocer la opinión del profesorado hacia la } \\
\text { diversidad la permanencia de diferentes alumnos } \\
\text { en sus aulas. }\end{array}$ & $\begin{array}{l}\text { Mixto } \\
\mathrm{n}=172\end{array}$ & $\begin{array}{l}\text { Cuestionario individual } \\
\text { Entrevistas abiertas }\end{array}$ \\
\hline $\begin{array}{l}\text { Cariaga \& } \\
\text { Grandon }(2021)\end{array}$ & Chile & $\begin{array}{l}\text { Describir las actitudes del profesorado de secundaria } \\
\text { hacia estudiantes que presentan un diagnóstico de } \\
\text { trastorno mental. }\end{array}$ & $\begin{array}{l}\text { Cuali } \\
\mathrm{n}=30\end{array}$ & $\begin{array}{l}\text { Entrevistas semi } \\
\text { estructuradas }\end{array}$ \\
\hline $\begin{array}{l}\text { Molinar \& } \\
\begin{array}{l}\text { Cervantes } \\
\text { (2021) }\end{array}\end{array}$ & México & $\begin{array}{l}\text { Analizar las actitudes de los docentes de educación } \\
\text { básica hacia los nińos con TDAH. }\end{array}$ & $\begin{array}{l}\text { Cuali } \\
\mathrm{n}=19\end{array}$ & Observación indirecta \\
\hline
\end{tabular}

\section{Sobre la metodología y el tipo de muestra}

En cuanto a la metodología utilizada, el 70\% de los trabajos revisados hace uso de técnicas cuantitativas, caracterizadas por la aplicación de adaptaciones de escalas de medición sobre las actitudes de profesionales hacia la inclusión o atención de estudiantes con discapacidad, a partir de una escala tipo Likert (Bermúdez \& Navarrete, 2020; Molinar \& Cervantes, 2020; Sanhueza et al., 2012; Polo y Aparicio, 2018; Víquez et al., 2020; Tárraga et al., 2020; Abellán, 2015; Angenscheidt \& Navarrete, 2017); también se utilizan cuestionarios adecuados a su contexto particular (Hernández \& Marchesi, 2021; Pegalajar \& Colmenero, 2017; Fernández-Rivas \& Espada-Mateos, 2017; Sevilla et al., 2018) y cuestionarios de creación propia de los autores (Montánchez, 2014; Novo-Corti et al., 2015). Un 15\% de los artículos analizados es de corte cualitativo con enfoques fenomenológico (Cariaga \& Grandon, 2021), descriptivo (Molinar \& Cervantes, 2021) y de corte etnográfico (Acosta \& Arráez, 2014). Estos trabajos se fundamentaron en las entrevistas semiestructuradas, la observación participante y la observación indirecta. Otro $10 \%$ de los artículos revisados desarrolló revi- siones de literatura (González \& Triana, 2018; Solís \& Borja, 2020); solo un artículo del total consultado, desarrolló una metodología mixta (Díaz \& Franco, 2010), aplicando la escala de actitudes (EAPROF), complementada con entrevistas focalizadas.

Las muestras son variadas, atendiendo a diferentes tipos de población, como docentes de básica primaria (Molinar \& Cervantes, 2020; Hernández \& Marchesi, 2021; Angenscheidt \& Navarrete, 2017; Bermúdez \& Navarrete, 2020), secundaria (Cariaga \& Grandon, 2021; Díaz \& Franco, 2010; Montánchez, 2014; Tárraga et al., 2020; Molinar \& Cervantes, 2021; Pegalajar \& Colmenero, 2017), preescolar (Polo \& Aparicio, 2018; Acosta \& Arráez, 2014), docentes universitarios (Novo-Corti et al., 2015), docentes de diferentes niveles escolares (Fernández-Rivas \& Espada-Mateos, 2017; Sevilla et al., 2018; Sanhueza et al., 2012; Víquez et al., 2020) y docentes en formación (Abellán, 2015). Estas muestras oscilan entre los 10 y los 600 participantes, encontrando 5 trabajos con muestras que superan los 200 participantes y 8 que se ubican por debajo de los 50 .

Del mismo modo, las procedencias de los estudios son variadas, con 6 investigaciones de España y 3 de Colombia, Chile y Uruguay. Por su parte, en 
Ecuador y México se encontraron 2 trabajos de cada uno y Venezuela, Costa Rica y Argentina presentaron un hallazgo. Igualmente, la distribución cronológica ubica 16 investigaciones entre los años 2015 y 2021 y 4 trabajos entre el 2010 y el 2014.

\section{Sobre los instrumentos de recolección de infor- mación}

Los instrumentos de recolección de información utilizados en los artículos revisados presentan algunas coincidencias en razón de los enfoques metodológicos utilizados por los autores; en esta medida, una de las herramientas más utilizadas en las investigaciones de corte cuantitativo es la "Escala de actitudes hacia las personas con discapacidad” (EAPD), creada por Verdugo et al. (1995), aplicada en 5 de los 15 trabajos en esta línea. La EAPD es una escala multidimensional desarrollada en el entorno español, que bajo una metodología tipo Likert analiza las actitudes que los profesionales en todas las áreas poseen con relación a la inclusión de personas con discapacidad. Esta escala cuenta con estudios de fiabilidad ( $\alpha$ de Cronbach $=.92)$ y un reconocimiento de validez por criterio de expertos en la materia (Víquez et al., 2020). La escala original consta de 37 ítems distribuidos en 5 niveles, los cuales evalúan: -Valoración de capacidades y limitaciones (VCL) -Reconocimiento/ negación de derechos (RND) -Implicación personal (IP) -Calificación genérica (CG) -Asunción de roles (AR) Al tratarse de un instrumento planteado en el entorno español, algunos autores realizaron procesos de adaptación, actualización y contextualización de conceptos y situaciones (Sanhueza et al., 2012; Abellán, 2015; Polo \& Aparicio, 2018; Bermúdez \& Navarrete, 2020), acompañados de una validación por expertos (Pegalajar \& Colmenero, 2017), ajustando los enunciados a las condiciones particulares de su población participante.

También se hace uso de otras escalas como la escala de actitudes (EAPROF) (Díaz \& Franco, 2010), la escala ASKAT (Molinar \& Cervantes, 2020) y una adaptación de la escala ORI (Opinions Relative to Integration of Students with Disabilities Scale)
(Tárraga et al., 2020). Montánchez (2014) elaboró un cuestionario con 79 ítems tipo Likert dividido en 2 partes y compuesto por las opciones "nada", "poco", "bastante" y "mucho". Novo-Corti et al. (2015) y Pegalajar \& Colmenero (2017) construyeron cuestionarios específicos para sus investigaciones con $20 \mathrm{y}$ 40 ítems, respectivamente; ambos en una escala tipo Likert de cinco opciones en las que 1 significa total desacuerdo y 5 totalmente de acuerdo.

En cuanto a las investigaciones de tipo cualitativo, se privilegian las entrevistas semi estructuradas (Acosta \& Arráez, 2014; Cariaga \& Grandon, 2021), la observación participante (Acosta \& Arráez, 2014) y la observación directa (Molinar \& Cervantes, 2021). Por su parte, Hernández \& Marchesi (2021) utilizan un cuestionario individual de 21 ítems tipo Likert de respuesta múltiple y entrevistas abiertas como técnicas para la recolección de información en su investigación de enfoque mixto.

\section{Sobre el tema central de la investigación}

En lo referente a las temáticas analizadas en los trabajos objeto de esta revisión, se hace énfasis en la actitud como elemento constitutivo de las investigaciones, sumado a las consideraciones de los docentes sobre la educación inclusiva y la presencia de estudiantes con discapacidad en el aula. En este marco, el abordaje de las actitudes docentes se centra en mayor medida en la discapacidad en general, aspecto en el que coincide el $77.8 \%$ de los trabajos revisados. Por otra parte, las actitudes docentes frente a la inclusión de estudiantes con trastorno por déficit de atención e hiperactividad (TDAH) es investigado en el 11\% de los trabajos (Molinar \& Cervantes, 2020; Molinar \& Cervantes, 2021). A su vez, el trastorno mental (Cariaga \& Grandon, 2021) y la discapacidad motriz (Acosta \& Arráez, 2014) son objeto de análisis en un trabajo cada uno.

\section{Un acercamiento a los conceptos clave}

Teniendo en cuenta que todos los trabajos revisados tienen coincidencia temática en cuanto al análisis de las 
actitudes de los docentes respecto de la inclusión de estudiantes con discapacidad en el aula, resulta evidente que uno de los temas fundamentales es la actitud, en general, y las actitudes centradas en el docente, de forma particular. Igualmente, se encuentran conceptos relevantes como educación inclusiva y discapacidad, los cuales serán definidos a continuación.

\section{Una mirada al concepto de actitudes docentes}

El tema de las actitudes ha sido estudiado en las ciencias sociales desde hace varias décadas, siendo la psicología y la educación unos de los campos que mayor atención han puesto en este aspecto (Molinar \& Cervantes, 2021). Así pues, las actitudes son definidas como una predisposición aprendida y organizada para pensar, sentir, actuar o reaccionar de manera favorable o desfavorable ante una situación (Bravo, 2013). Las actitudes están conformadas por componentes de tipo afectivo, cognitivo y conductual (Molinar \& Cervantes, 2021), relacionados con las emociones y los sentimientos generados por la situación, el contexto o las personas.

El componente afectivo se caracteriza por una fuerte carga emocional, que genera sentimientos positivos o negativos que afectan la manera en que una persona reacciona ante cierto tipo de estímulos (Briñol et al., 2008; Morales, 2007). El elemento cognitivo se refiere a las ideas previas sobre un aspecto determinado que resultan del aprendizaje individual o colectivo, las cuales se forman mediante la interacción y el reconocimiento de experiencias particulares en torno a la situación, el concepto o el sujeto objeto de la actitud (Briñol et al., 2008). El aspecto conductual responde a las reacciones concretas que tienen las personas, las cuales se fundamentan en lo afectivo y lo cognitivo, para generar actos más o menos estables acerca de la misma situación (Molinar \& Cervantes, 2021). Por consiguiente, las actitudes docentes hacen referencia a las formas en que los maestros asumen, reaccionan e interactúan con las situaciones propias del contexto educativo y social. Estas actitudes se reflejan en los pensamientos, los actos, las creencias y los discursos del profesorado; se forman a lo largo de su experiencia personal y laboral (Verdugo, 2002) y se manifiestan en sus intervenciones cotidianas, tanto dentro como fuera de las instituciones educativas (Beltrán et al., 2007; Sandoval, 2009).

\section{La educación inclusiva y la discapacidad}

Hablar de educación inclusiva y de discapacidad implica elaborar un discurso amplio y pormenorizado que escapa al objetivo de esta revisión; así pues, los conceptos presentados a continuación constituyen un resumen, por demás rápido, de estos términos. Bajo el término de educación inclusiva se conjugan todas las formas de atender las particularidades de cada estudiante, como parte de un sistema educativo acondicionado para responder a la diversidad y acoger la diferencia, respetando las capacidades y posibilidades individuales, ofreciendo programas formativos oportunos y de calidad (UNESCO, 2005; Booth \& Ainscow, 2011; Acosta \& Arráez, 2014; Darretxe et al., 2020). La discapacidad, por su parte, es definida como una situación de la persona que debido a sus condiciones físicas, mentales, intelectuales y sensoriales duraderas, enfrenta barreras de acceso, participación social o interacción con el entorno (Organización de las Naciones Unidas, 2006; Real Academia Española, 2021).

\section{Discusión y conclusiones}

Las investigaciones observadas como parte de este trabajo muestran resultados ambivalentes en cuanto a las actitudes del profesorado con relación a la educación inclusiva y la atención a los estudiantes con discapacidad en el aula regular. Esto resulta natural puesto que el análisis de las actitudes docentes continúa siendo un tema sumamente complejo de abordar y presenta especificidades que cambian dependiendo del entorno (Di nardo et al., 2014). Por ende, las actitudes favorables o desfavorables sobre esta situación requieren de un análisis pormenorizado de cada contexto (Bravo, 2013). A pesar de esto, la literatura revisada muestra una tendencia hacia las actitudes positivas (Polo \& 
Aparicio, 2018; Tárraga et al., 2020; Angenscheidt \& Navarrete, 2017; González \& Triana, 2018; Sanhueza et al., 2012; Abellán, 2015; Solís \& Borja, 2020), las cuales se presentan en mayor medida en los docentes jóvenes (Tárraga et al., 2020), debido a que parte de su formación profesional incluyó algún tipo de acercamiento al campo de la discapacidad, ya sea desde un punto de vista teórico o, en algunos casos, con experiencias vivenciales (Tárraga et al., 2020). Asimismo, los maestros en formación muestran una actitud más positiva hacia los procesos de educación inclusiva que otros maestros con mayor experiencia (Vélez, 2013). Estos resultados se asemejan a los hallados en las investigaciones de Santiago \& Marrero (2003) y Clavijo et al. (2016), quienes encontraron que los docentes más jóvenes suelen presentar actitudes más favorables ante los estudiantes con discapacidad, en comparación con sus colegas de mayor recorrido. No obstante, el trabajo de Angenscheidt \& Navarrete (2017) contradice estos resultados, pues, aunque como parte de su investigación encontraron que la mayoría de los participantes tenían actitudes favorables, también concluyeron que los docentes con mayor experiencia tienen una actitud más positiva frente a la educación inclusiva. En cuanto al nivel escolar, Angenscheidt \& Navarrete (2017) concluyeron que no existe una diferencia significativa en el tipo de actitudes de los maestros con relación al ciclo académico que imparten.

De igual manera, las actitudes del profesorado con respecto a la inclusión de estudiantes con discapacidad en sus aulas varían de acuerdo con algunas características particulares que están relacionadas con la formación del docente, su experiencia en el acompañamiento de esta población y con los recursos que tanto el Estado - de forma macro- como su institución -de forma micro- puedan ofrecerle (Soto, 2007; Cardona, 2006). En esta medida, los resultados de algunas investigaciones muestran que los docentes que presentan actitudes desfavorables con respecto a la discapacidad tienen poca formación sobre el tema (Cariaga $\&$ Grandon, 2021), manifestando su actitud negativa en forma de sobreprotección, lástima o rechazo hacia los estudiantes en esta condición (Soto, 2007).
En esa misma línea, la formación profesional y los procesos de capacitación que reciben los docentes se convierten en un elemento determinante para el establecimiento de sus actitudes. Al tenor, los resultados de la investigación de Sánchez \& Carrión (2002) afirman que las actitudes negativas están asociadas a la falta de formación adecuada en la materia, lo que implica que los docentes desconocen las estrategias metodológicas apropiadas para adaptar su trabajo a las necesidades de los estudiantes con discapacidad. Estos resultados son coincidentes con los obtenidos en otras investigaciones de este corte, en las que se puede observar que una de las causas más comunes de las actitudes desfavorables del colectivo docente hacia la inclusión de estudiantes con discapacidad está originada en su poca formación y su escaso conocimiento al respecto (Tenorio, 2011; Vélez, 2013; Pegalajar \& Colmenero, 2017). En cuanto a las limitaciones de esta revisión, se encuentra la restricción del lenguaje, pues al abordar textos publicados únicamente en lengua castellana, se deja por fuera una amplia gama de publicaciones y estudios relacionados. En contraposición, este trabajo y sus condiciones particulares pueden ofrecer una visión actualizada sobre el estado de este tema y de algunas de las metodologías bajo las cuales ha sido abordado en el contexto hispanoparlante.

En conclusión, las publicaciones académicas sobre las actitudes de los docentes frente a los procesos de inclusión de estudiantes con discapacidad han venido aumentando en los últimos años; aun así, la literatura disponible es escasa (Solís \& Borja, 2020), lo que implica que este es un campo de estudio que requiere de la implementación de investigaciones encaminadas al reconocimiento del tipo de actitud de los docentes, sus causas y los comportamientos que generan, sumado a las posibles estrategias para modificar las actitudes menos favorables, lo cual redundará de forma directa en el beneficio de los estudiantes con discapacidad y en los procesos educativos de toda la población estudiantil.

En suma, los resultados obtenidos en esta revisión ofrecen conclusiones interesantes que permiten establecer líneas de investigación relevantes para 
profundizar en este campo de estudio. Entre ellas, se encuentran posibles comparaciones entre los procesos de formación a nivel universitario actuales y los que se llevaban a cabo hace algunas décadas, puesto que, como se pudo observar, la formación y la capacitación de los docentes influye de manera directa en sus actitudes frente a la educación inclusiva y a la atención a los estudiantes con discapacidad.

Partiendo de lo anterior, los procesos de formación y capacitación docente podrían fortalecer las líneas de trabajo relacionadas con el estudio de la normatividad nacional e internacional vigente respecto de la atención al estudiantado con discapacidad, toda vez que estos lineamientos representan la base sobre la cual se establecen las diversas prácticas pedagógicas, tanto en las instituciones privadas como en las de naturaleza pública. Igualmente, se deben fomentar prácticas en las que se involucre de forma directa e intencionada la atención y el acompańamiento a estudiantes con discapacidad. De esta manera, se posibilita el intercambio de experiencias y la toma de conciencia sobre las necesidades de esta población y la forma en que los docentes aportan al desarrollo de procesos académicos coherentes y significativos, en el marco de la educación inclusiva.

Por otra parte, los docentes en ejercicio requieren de jornadas de actualización conceptual y metodológica al respecto, ya que la educación inclusiva se constituye como un campo dinámico y en constante evolución, lo que genera que algunas de las concepciones sobre la atención de los estudiantes con discapacidad en las aulas regulares hayan cambiado sustancialmente en los últimos años, haciendo que los docentes con más años de egreso de las facultades desconozcan esos cambios.

En resumen, los docentes desempeñan un rol fundamental en los procesos de inclusión de estudiantes con discapacidad; además, son ellos los encargados de materializar las políticas, los acuerdos e iniciativas de las entidades gubernamentales y de poner en contexto las propuestas de organizaciones internacionales en materia de inclusión y atención a la diversidad. Finalmente, resulta llamativa la poca presencia de investigaciones que dispongan de una metodología mixta, postura que podría arrojar resultados significativos en razón de las posibilidades que la integración de las características del trabajo cuantitativo y cualitativo pueden ofrecer para el análisis de las situaciones en diversos contextos.

\section{Referencias}

Abellán, J. (2015). Actitudes hacia la discapacidad mostradas por los futuros maestros de Educación Física. Sportis. Scientific Journal of School Sport, Physical Education and Psychomotricity, 1(3), 207-219. https://doi.org/10.17979/ sportis.2015.1.3.1414

Acosta, A. \& Arráez, T. (2014). Actitud del docente de educación inicial y primaria ante la inclusión escolar de las personas con discapacidad motora. Revista de Investigación, 83(38), 135-154. https://dialnet.unirioja.es/servlet/articulo?codigo $=6324729$

Amor, A.M., Fernández, M., Verdugo, M. Ángel., Aza, A., \& Calvo, M.I. (2021). Towards the fulfillment of the right to inclusive education for students with intellectual and developmental disabilities. Framework for action. Education Sciences \& Amp: Society - Open Access, 12(1). https://doi.org/10.3280/ess1-2021oa11471

Angenscheidt, L. \& Navarrete, I. (2017). Actitudes de los docentes acerca de la educación inclusiva. Ciencias Psicológicas, 11(2), 233-243. https://www.redalyc.org/articulo.oa? id= 459553539013

Baez, M. \& D’ Ottavio, M. E. (2020). La diversidad en el aula: el desafío de interpretar la singularidad de los procesos de alfabetización inicial. Ciencia y Educación, 3(3), 31-40. https://doi. org/10.22206/cyed.2019.v3i3.pp31-40

Beltrán, L., Agudelo, K., Pérez, C., Cortés, L., Úsuga, J., Garavito, L. \& Betancur, L. (2007). Formación docente: una propuesta para promover prácticas pedagógicas inclusivas. Revista Educación y pedagogía, 19(47). https://revistas.udea.edu.co/index. $\mathrm{php} /$ revistaeyp/article/view/6679 
Beltrán, Y., Martínez, Y. \& Vargas, Á. (2015). El sistema educativo colombiano en el camino hacia la inclusión. Avances y retos. Educación y Educadores, 18(1), 132-140. 10.5294/edu.2015.18.1.4

Bermúdez, M. \& Navarrete, I. (2020). Actitudes de los maestros ante la inclusión de alumnos con discapacidad. Ciencias Psicológicas, 14(1), e-2107. https://doi.org/10.22235/cp.v14i1.2107

Blanco, R. (2006). La equidad y la inclusión social: uno de los desafíos de la educación y la escuela de hoy. Revista Electrónica Iberoamericana sobre Calidad, Eficacia y Cambio en Educación, 4(3), 1-15. https://www.redalyc.org/pdf/ 551/55140302.pdf.

Booth, T. \& Ainscow, M. (2011). Índex para la inclusión. Guía para la evaluación y mejora de la educación inclusiva. Consorcio Universitario para la Educación Inclusiva. https://doi. org/10.18172/con.514

Bravo, L. (2013). Percepciones y opiniones hacia la educación inclusiva del

profesorado y de los equipos directivos de los centros educativos de la dirección regional de Cartago en Costa Rica [Tesis doctoral, Universidad de Alicante]. https://eprints.ucm.es/id/eprint/44512/1/ T39229.pdf

Briñol, P., Falces, C. \& Becerra, A. (2008). Actitudes. Psicología social, 3, 457-490. https:/www. researchgate.net/publication/271838160_ Actitudes.

Cardona, M. (2006). Diversidad y educación inclusiva: enfoques metodológicos y estrategias para una enseñanza colaborativa. Pearson-PrenticeHall.

Cariaga, C. \& Grandon, P. (2021). Actitudes del profesorado de secundaria hacia estudiantes con diagnóstico de trastorno mental en colegios de la provincia de Concepción y Diguillín: Un estudio cualitativo. Revista de Investigación Educativa, 39(1), 283-298. http://dx.doi. org/10.6018/rie.428681

Clavijo, R., López, C., Cedillo, C., Mora, C. \& Ortiz, W. (2016). Actitudes docentes hacia la educa- ción inclusiva en Cuenca. Maskana, Cuenca, 7(1) 13-22. 10.18537/mskn.07.01.02

Córdoba-Andrade, L., Muriel-Acuña, I. \& Enciso-Luna, J. E. (2021). Representaciones sociales de la discapacidad en una comunidad universitaria de Ibagué, Colombia, mediante las redes de asociaciones. Revista Colombiana de Ciencias Sociales, 12(1), 114-139. https://doi. org/10.21501/22161201.3364

Darretxe, L., Beloki, B. \& Remiro, A. (2020). Sociedad y escuela que deseamos: la dialéctica entre inclusión y exclusión social. Ciencia y Educación, 4(1), 71-81. https://doi.org/10.22206/cyed.2020. v4i1.pp71-81

Díaz, O. \& Franco, F. (2010). Percepción y actitudes hacia la inclusión educativa de los docentes de Soledad, Atlántico (Colombia), 2008. Zona Próxima, (12), 12-39. https://www.redalyc.org/ articulo.oa?id=85316155002

Di Nardo, M., Kudláček, M., Tafuri, D., \& Sklenaříková, J. (2014). Attitudes of preservice physical educators toward individuals with disabilities at University Parthenope of Napoli. Acta Gymnica, 44(4), 211-221. https://doi. org/10.5507/ag.2014.022

Fernández-Rivas, M. ${ }^{a}$ \& Espada-Mateos, M.a (2017). Evaluación de las dificultades de llevar a cabo la atención a la diversidad por parte del profesorado de Educación Física. Sportis Sci J, 3(3), 542-553. https://doi.org/10.17979/ sportis.2017.3.3.2051

González, Y. \& Triana, D. (2018). Actitudes de los docentes frente la inclusión de estudiantes con necesidades educativas especiales. Educación y Educadores, 21(2). 200-218. 10.5294/edu. 2018.21.2.2

Hernández, L. \& Marchesi, Á. (2021). Actitudes de los maestros ante la inclusión educativa en Colombia, Guatemala y España. Ciencia y Educación, 5(1), 7-24. https://doi.org/10.22206/ cyed.2021.v5i1.pp7-24 Latorre, L. (2013). La educación inclusiva en docentes en formación: su evaluación a partir de la teoría de facetas. Folios, 
37, 95-113. Ministerio de Educación Nacional. (2007). Educación para todos. Al Tablero, (43), 1. http://www.mineducacion.gov.co/1621/ article-141881.html Molinar, J. \& Cervantes, A. (2020). Comparación de los conocimientos y actitudes del profesorado de educación básica hacia el alumno con el trastorno por déficit de atención e hiperactividad TDAH en Argentina y México. REXE Revista de Estudios y Experiencias en Educación, 19(40), 171-181. https://doi. org/10.21703/rexe.20201940molinar9

Molinar, J. \& Cervantes, A. (2021). Actitudes percibidas en el discurso narrativo docente hacia el alumnado con TDAH. Un estudio de observación indirecta. REXE Revista de Estudios y Experiencias en Educación, 20(42), 87-100. https:// doi.org/10.21703/rexe.20212042molinar5

Montánchez, M. (2014). Las actitudes, conocimientos y prácticas de los docentes de la ciudad de esmeraldas ante la inclusión educativa: un estudio exploratorio. Espacio $I+D$ Innovación más Desarrollo, 3(5), 114-140. 10.31644/ IMASD.5.2014.a06

Mora, A., Córdoba, L. \& Rodríguez, C. (2020). Resignificación de las creencias familiares para la autodeterminación del adulto con discapacidad intelectual. Revista latinoamericana en discapacidad, sociedad y derechos humanos, 4(1), 83-104. http://redcdpd.net/revista/index.php/revista/ article/view/180

Morales, F. (2007). Psicología Social. Tercera edición. Mc Graw Hill/Interamericana de España.

Morales, I. (2017). Calidad de vida en el trastorno por déficit de atención con hiperactividad: disfunción ejecutiva y comorbilidad [Tesis doctoral, Universidad Complutense de Madrid]. https://eprints. ucm.es/id/eprint/44512/1/T39229.pdf.

Novo-Corti, I., Muñoz-Cantero, J. \& Calvo-Babío, N. (2015). Los futuros docentes y su actitud hacia la inclusión de personas con discapacidad: una perspectiva de género. Anales de Psicología, 31(1), 155-171. https://dx.doi.org/10.6018/ analesps.31.1.163631
Organización de las Naciones Unidas (ONU). (2006). Convención sobre los Derechos de las Personas con Discapacidad y Protocolo Facultativo. http:// www.un.org/disabilities/documents/convention/convoptprot-s.pdf

Pegalajar, M. \& Colmenero, M. (2017). Actitudes y formación docente hacia la inclusión en Educación Secundaria Obligatoria. Revista Electrónica de Investigación Educativa, 19(1), 84-97. https:// doi.org/10.24320/redie.2017.19.1.765

Polo, M. \& Aparicio, M. (2018). Primeros pasos hacia la inclusión: Actitudes hacia la discapacidad de docentes en educación infantil. Revista de Investigación Educativa, 36(2), 365-379. http:// dx.doi.org/10.6018/rie.36.2.279281

Real Academia Española. (2021). Discapacidad. Diccionario de la lengua española. https://dle. rae.es/discapacidad? $\mathrm{m}=$ form

Sánchez, A. \& Carrión, J. (2002). Una aproximación a la investigación en educación especial. Revista de Educación, 327, 225-247. https:// www.mecd.gob.es/dctm/revista-de-educacion/ articulos327/re3271410520.pdf?documentI$\mathrm{d}=0901 \mathrm{e} 72 \mathrm{~b} 812598 \mathrm{~b} 5$

Sánchez-Gómez, V., López, M., Amor, A. \& Verdugo, A. (2020). Apoyos para la Calidad de Vida de Escolares con y sin Discapacidad: Revisión de Literatura. Revista Internacional de Educación para la Justicia Social, 9(2), 327-349. https:// doi.org/10.15366/riejs2020.9.2.016

Sandoval, L. (2009). Las interacciones sociales que se desarrollan en los salones de clase y su relación con la práctica pedagógica que realiza el docente en el aula. Revista de posgrado y sociedad, 9(2), 32-57. https://dialnet.unirioja.es/servlet/articulo? codigo=3662261.

Sanhueza, S., Granada, M. \& Bravo, L. (2012). Actitudes del profesorado de chile y costa rica hacia la inclusión educativa. Cadernos de Pesquisa, 42,(146). https://www.scielo.br/j/ cp/a/qzgV4Hm9XV9Tbsc7hDG37nw/?lan$\mathrm{g}=\mathrm{es} \&$ format $=\mathrm{pd}$ 
Santiago, O. \& Marrero, G. (2003). Actitudes hacia las personas con discapacidad en profesionales del primer ciclo de Educación Infantil. Anuario de Filosofía, Psicología y Sociología, 6, 131-158. Sevilla, D., Martín, M. \& Jenaro, C. (2018). Actitud del docente hacia la educación inclusiva y hacia los estudiantes con necesidades educativas especiales. Innovación educativa (México, DF), 18(78), 115-141. http:// www.scielo.org. $\mathrm{mx} / \mathrm{scielo}$.php?script $=$ sci_ arttext\&pid=S1665-26732018000300115\&l$\mathrm{ng}=\mathrm{es} \& \mathrm{t} \operatorname{lng}=\mathrm{es}$.

Slee, R. (2018). Inclusive education: from policy to school implementation. In Clark, C., Dyson, A. \& Millward, Alan (Ed.). Towards inclusive schools (pp. 30-41). Routledge.

Solís, P. \& Borja, V. (2020). Profesorado de educación física: sus actitudes y creencias hacia la discapacidad. Revisión de la literatura como punto de partida. VIREF Revista De Educación Física, 9(3), 87-103. https://revistas.udea.edu.co/index. $\mathrm{php} /$ viref/article/view/340609

Soto, R. (2007). Actitud docente de la Universidad de Costa Rica hacia los (as) estudiantes con discapacidad de la Universidad. Revista Educación, 31(1), 11-42. https:/www.redalyc.org/articulo. oa? id=44031 102

Tárraga-Mínguez, R., Vélez-Calvo, X., Pastor-Cerezuela, G. \& Fernández-Andrés, M. (2020). Las actitudes del profesorado de educación primaria hacia la educación inclusiva en Ecuador. Educação e Pesquisa, 46, 1-17. https://doi. org/10.1590/S1678-4634202046229504

Tenorio, S. (2011). Formación inicial docente y necesidades educativas especiales. Estudios Pedagógicos, 37(2), 249-265. http://dx.doi.org/10.4067/ S0718-07052011000200015
UNESCO. (1994). Declaración de Salamanca y marco de acción para las necesidades educativas especiales. UNESCO. http://www.unesco.org/ education/pdf/SALAMA_S.PDF

UNESCO (2005). Informe de seguimiento de la educación para todos, 2006. La alfabetización, un factor vital. UNESCO.

Vélez, L. (2013). La educación inclusiva en docentes en formación: su evaluación a partir de la teoría de facetas. Folios. Segunda Época, 37, 95-113. http://www.scielo.org.co/pdf/folios/n37/ n37a07.pdf

Verdugo, M. (2002). Persona con discapacidad: Perspectivas psicopedagógicas y rehabilitadoras. Siglo xxi. Verdugo, M., Jenaro, C. \& Arias, B. (1995). Actitudes sociales y profesionales hacia las personas con discapacidad: Estrategias de evaluación e intervención. En M. Á. Verdugo (Ed.), Personas con discapacidad: perspectivas psicopedagógicas y rehabilitadoras (pp. 79-143). Siglo XXI.

Verdugo, M., Córdoba Andrade, L. y Rodríguez Aguilella, A. (2020). Calidad de vida en familias de personas con discapacidad. Pensando Psicología, 16(1), 1-23. https://doi.org/10.16925/23823984.2020.01.01

Víquez, F., Quirós, S., Rodríguez-Méndez, D. \& Solano, L. (2020). La inclusión de personas con discapacidad en una escuela multideportiva: efecto de las actitudes hacia la discapacidad en niños, niñas, jóvenes, padres, madres y personal de instrucción. MHSalud, 17(2), 1-16. https://doi.org/10.15359/mhs.17-2.3 